\title{
Assessment of coronary artery disease during hospitalization for cancer treatment
}

\author{
Simone M. Mrotzek ${ }^{1}$. Alessia Lena ${ }^{2,3,4,5} \cdot$ Sara Hadzibegovic ${ }^{2,3,4,5} \cdot$ Ria Ludwig $^{1} \cdot$ Fadi Al-Rashid $^{1} \cdot$ Amir A. Mahabadi ${ }^{1}$. \\ Raluca I. Mincu ${ }^{1}$ - Lars Michel ${ }^{1}$ - Laura Johannsen ${ }^{1} \cdot$ Lena Hinrichs $^{1} \cdot$ Martin Schuler $^{6,7}$. Ulrich Keller ${ }^{8,9,10}$. \\ Stefan D. Anker ${ }^{2,4,5}$. Ulf Landmesser ${ }^{3,4,5}$. Tienush Rassaf ${ }^{1} \cdot$ Markus S. Anker $^{2,3,4,5} \cdot$ Matthias Totzeck $^{1}$
}

Received: 19 May 2020 / Accepted: 23 July 2020 / Published online: 2 August 2020

(C) The Author(s) 2020

\begin{abstract}
Background With improvement of cancer-specific survival, comorbidities and treatment-related side effects, particularly cardiovascular toxicities, need close attention. The aim of the present study was to evaluate clinical characteristics and outcomes of cancer patients requiring coronary angiography during inpatient care.

Methods We performed a retrospective analysis of patients hospitalized between 02/2011 and 02/2018 in our two university hospital cancer centers. From a cohort of 60,676 cancer patients, we identified 153 patients $(65.7 \pm 11.6$ years, $73.2 \%$ male), who underwent coronary angiography and were eligible for analysis. These were compared to a control group of 153 noncancer patients pair-matched with respect to age, sex, and indication for catheterization.

Results Cancer patients presented in $66 \%$ with an acute coronary syndrome (ACS). The most prevalent cancer entities were lymphoma (19\%) and lung cancer (18.3\%). The rate of primary percutaneous coronary interventions (PCI) was significantly lower in the cancer cohort ( $40.5 \%$ vs. $53.6 \%, p=0.029)$, although manifestation of coronary artery disease (CAD) and PCI results were comparable (SYNergy between PCI with TAXus and cardiac surgery (SYNTAX)-score, delta pre- and postPCI -9.8 vs. $-8.0, p=0.2)$. Mortality was remarkably high in cancer patients ( 1 -year mortality $46 \%$ vs. $8 \%$ in non-cancer patients, $p<0.001)$, particularly with troponin-positive ACS (5-year mortality $71 \%)$.

Conclusion Strategies to effectively control cardiovascular risks in cancer patients are needed. Additionally, suspected CAD in cancer patients should not prevent prompt diagnostic clarification and optimal revascularization as PCI results in cancer patients are comparable to non-cancer patients and occurrence of troponin-positive ACS leads to a significantly increased risk of mortality.
\end{abstract}

\section{Graphic Abstract}

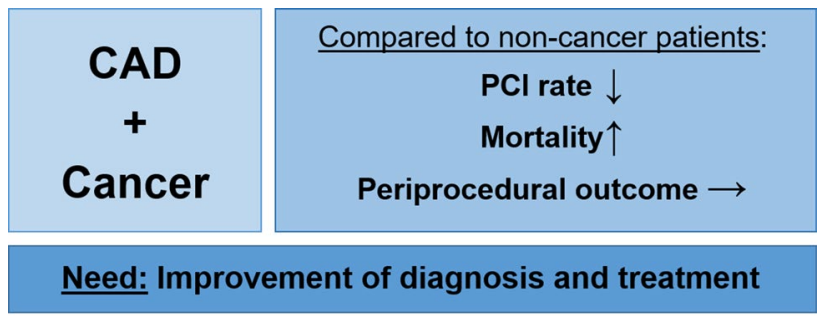

Keywords Coronary artery disease $\cdot$ Acute coronary syndrome $\cdot$ Cancer $\cdot$ Cardio-oncology $\cdot$ Cardiotoxicity

Electronic supplementary material The online version of this article (https://doi.org/10.1007/s00392-020-01719-5) contains supplementary material, which is available to authorized users.

Extended author information available on the last page of the article 


\section{Introduction}

Cardiovascular diseases and cancer are the leading causes of death in western countries [1]. Cancer and cancer therapy may accelerate or induce coronary artery disease (CAD) and provoke acute coronary syndromes (ACS) [2]. Underlying mechanisms, treatment characteristics, and outcomes are incompletely characterized [3]. Additionally, common risk factors contribute to a higher prevalence of CAD in cancer patients [4]. Until now, guidelines for the treatment and management of patients with ACS and chronic coronary syndromes are not validated for cancer patients [5-7]. Uncertainties particularly pertain to the diagnosis of an ACS as biomarkers have been tested in only a limited number of cancer patients [8]. Troponin is as a well-established specific and sensitive marker of myocardial injury and infarction with high diagnostic and prognostic value for ACS patients [9-11]. In cancer patients, troponin release may additionally relate to anticancer treatments (e.g., anthracycline-related cardiotoxicity), which must be differentiated from an ACS [12-15]. Previous studies on patients with cancer and CAD showed that this cohort may be undertreated from the clinical and interventional point of view $[16,17]$. Low platelet counts, bleeding complications, cancer surgery, percutaneous coronary intervention (PCI), and antiplatelet therapy represent major challenges in the treatment of cancer patients with CAD [18-20]. Decision algorithms for coronary angiography and peri-interventional management pathways for cancer patients are incompletely defined $[3,21]$. Therefore, the objectives of our study were to evaluate clinical features and outcomes of this particular cancer cohort.

\section{Methods}

A retrospective, descriptive data analysis of patients of the West German Cancer Center, University Hospital Essen, and the Department of Hematology, Oncology and Tumor Immunology, Charité Campus Benjamin Franklin, Berlin (two of 13 comprehensive cancer centers in Germany), who were hospitalized between 02/2011 and $02 / 2018$, was performed. In total, 60,676 patients were screened and all discharged patients with a clinical procedure code of coronary angiography (OPS 1-275, German adaption of the International Classification of Procedures in Medicine of the World Health Organization, version 2018) were reviewed. Screening identified 210 potentially eligible patients. After exclusion of 57 cases because of double count, missing data, or other reason for cardiac catheterization (e.g., cardiac biopsy and right heart catheterization), 153 patients treated at the cardiology departments of both hospitals [Department of Cardiology and Vascular Medicine, West German Heart and Vascular Center, University Hospital Essen and Department of Cardiology, Charité Campus Benjamin Franklin (CBF), Berlin, Germany] were eligible for further analyses. The study flowchart is illustrated in Fig. 1. Pair matching was performed using a "closest neighbor greedy" algorithm [22], to match each cancer and cancer-naive patient with respect to age ( \pm 5 years), sex (male/female), and indication for coronary angiography. Patients were divided in four groups: ST-segment elevation myocardial infarction (STEMI), non-STEMI (NSTEMI), unstable angina, or stable angina (including patients with exertional angina pectoris and angina correlate). The non-cancer (control) cohort was randomly derived from patients hospitalized in the West German Heart and Vascular Center Essen or Department of Cardiology, Charité Campus Benjamin Franklin Berlin during 2017 and 2018 by reviewing reporting lists. The study was approved by the local ethics committees.

Statistical analyses of patient characteristics were conducted with respect to cancer entity, concurrent therapy, previous treatments, co-medication, and the individual patient's medical history, especially known CAD and cardiovascular risk factors. Laboratory findings were compared as documented on the day before or day of performed coronary angiography. Bleeding complications in cancer and non-cancer patients have been analyzed referring to BARC (Bleeding Academic Research Consortium) definition [23]. A detailed assessment of cardiac catheter examination results was additionally performed. SYNTAX (SYNergy between percutaneous coronary intervention (PCI) with TAXus and cardiac surgery)-score calculation as a validated tool for risk stratification and revascularization strategy selection in patients with complex CAD [24, 25] was independently performed by two investigators. Patients with coronary artery bypasses were excluded [25]. SYNTAX-score values were classified according to the common standards in the lowest tertile $(\leq 22)$, intermediate tertile (23-32), and highest tertile $(\geq 33)$ [25]. PCI result was assessed by the residual SYNTAX-score and delta between pre- and post-PCI SYNTAX-score [26]. All-cause mortality of cancer patients was analyzed as documented in the database of our centers for a follow-up of up to 5 years after coronary angiography and 1 year for non-cancer patients.

Quantitative variables were checked for normal distribution and expressed as mean \pm standard error of the mean (SEM) or as median and interquartile range (IQR, Q1 25\% to Q3 75\%). Dichotomous categorical variables were expressed as numeric values and percentages. For comparison of quantitative variables, an unpaired Student's t test or Mann-Whitney $U$ test (for non-normal distributed variables) was used. 


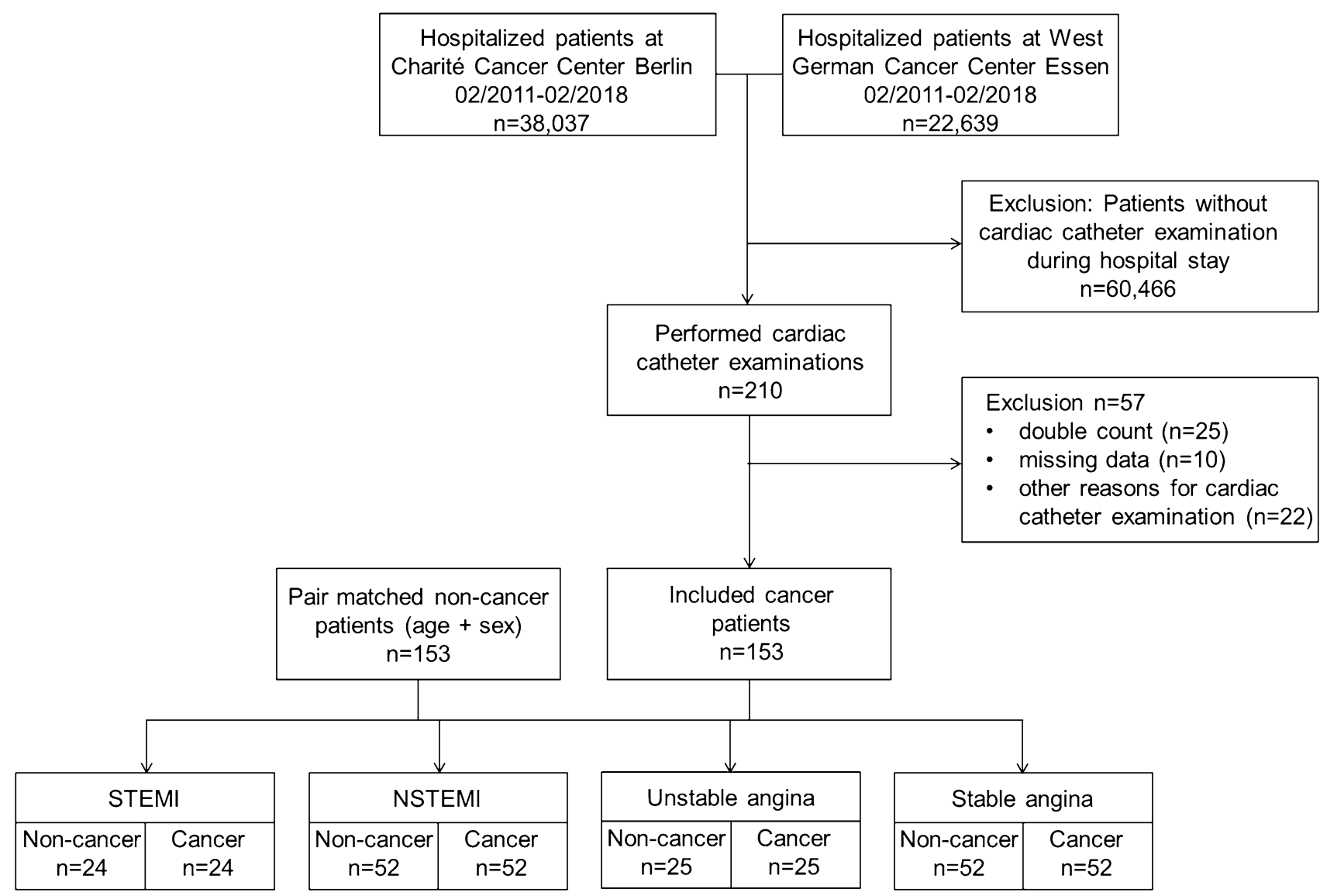

Fig. 1 Flowchart of the study design. Medical data banks of patients hospitalized in the West German Cancer Center, University Hospital Essen and Charité Campus Benjamin Franklin Berlin between $02 / 2011$ and 02/2108 were screened for performed coronary angiography using the clinical procedure code (OPS). From 60,676 patients, 210 patients were identified. 57 patients were excluded. Included

Dichotomous categorical variables were analyzed using Chi square test and Fisher's exact test. Logistic regression analysis was used to determine the association of individual patients' risk factors with PCI, presence of a multivessel CAD or 1-year mortality. Multivariable analyses were performed using the following adjustment sets: (1) age, gender, BMI, hypertension, diabetes, dyslipidemia, smoking, and previously known CAD adjusted, and (2) for cancer patients: age, gender, BMI, hypertension, diabetes, dyslipidemia, smoking, known CAD, metastatic disease, anthracycline chemotherapy, 5- fluorouracil chemotherapy, and chest irradiation adjusted, and (3) for 1-year mortality: age, gender, BMI, hypertension, diabetes, dyslipidemia, smoking, troponin-positive ACS, hemoglobin value, creatinine value, and platelet count. Kaplan-Meier procedure was calculated using log-rank tests (Mantel Cox) and Cox proportional hazard models to compare the survival curves of cancer and non-cancer patients as well as cancer patients with troponin-positive ACS (STEMI/NSTEMI) or troponin-negative cancer patients were divided by indication for coronary angiography: ST-segment elevation myocardial infarction (STEMI), non-STEMI (NSTEMI), unstable angina, and stable angina (including angina equivalents). Cancer patients were pair-matched with 153 non-cancer patients with respect to age, sex, and reason for coronary angiography

presentation (unstable/stable angina). Five-year survival of cancer patients was additionally analyzed using data from the Surveillance, Epidemiology, and End Results Program (SEER) from the US National Cancer Institute. Cancer Statistics Review between 1975 and 2013 was released April 15, 2020 (https://seer.cancer.gov/archive/csr/1975_2013/ results_merged/topic_survival.pdf) [27]. Listed survival rates from the SEER Cancer Statistics Review are calculated from 2006-2012. Patient's data were matched to our study cancer cohort regarding sex, age, cancer entity, and the presence of a metastatic (distant) cancer disease. The statistical 5-year survival rate for each cancer patient from our study was determined from the SEER database and included as a part of the total 5-year survival rate for comparison with our cancer cohort. This was not possible for eight of our cancer patients due to missing information on the cancer entity within the SEER database (e.g., choroidal melanoma, choriocarcinoma) or not well-defined cancer entity (e.g., cancer of unknown origin). 
Data were analyzed using IBM SPSS Statistics 25. Statistical significance was defined by a $\mathrm{p}$ value of $<0.05$.

\section{Results}

In the present study, 153 cancer patients fulfilled the inclusion criteria and were pair-matched to 153 control patients without a history of cancer. Baseline characteristics of the study population are presented in Table 1. Differences between the groups regarding cardiovascular risk factors and medical history were found with a higher body mass index in non-cancer group $\left(27.3 \mathrm{~kg} / \mathrm{m}^{2}\right.$ vs. $\left.26.0 \mathrm{~kg} / \mathrm{m}^{2}, p=0.014\right)$ and higher rates of documented dyslipidemia (66\% vs. $48.4 \%$, $p=0.003$ ). Moreover, cancer patients had a higher occurrence of chronic obstructive pulmonary disease (11.8\% vs.
$20.9 \%, p=0.044)$. Laboratory findings showed significantly lower hemoglobin value in cancer patients $(13.62 \mathrm{~g} / \mathrm{dl} \mathrm{vs}$. $10.83 \mathrm{~g} / \mathrm{dl}, p<0.001)$, as well as a trend to lower platelet counts $(236 / \mathrm{nl}$ vs. $219 / \mathrm{nl}, p=0.06)$. Previous treatment with acetylsalicylic acid or lipid-lowering therapy with statins was significantly less frequent in the cancer group $(p=0.001$ and $p<0.001$, respectively). Figure 2 illustrates the distribution of cancer entities in our study population. Lymphoma was the most common cancer entity with 29 patients (19\%), followed by lung cancer (28 patients, $18.3 \%$ ) and gastrointestinal cancer (23 patients, $15 \%$ ).

Results of cardiac catheterization are summarized in Table 2. Obstructive CAD was not detectable in $26.1 \%$ of the non-cancer and in $28.8 \%$ of the cancer patients $(p=0.205)$. Figure 3 a shows the occurrence of CAD and distinguishes between 1-, 2-, and 3-vessel-disease with no significant

Table 1 Baseline characteristics

\begin{tabular}{|c|c|c|c|}
\hline & Non-cancer $(n=153)$ & Cancer $(n=153)$ & $p$ value \\
\hline Age, years & $65.8( \pm 11.8)$ & $65.7( \pm 11.6)$ & 0.949 \\
\hline Male sex, $n(\%)$ & $112(73.2)$ & $112(73.2)$ & 1.000 \\
\hline BMI, $\mathrm{kg} / \mathrm{m}^{2}$ & $27.3( \pm 4.6)$ & $26.0( \pm 4.8)$ & $0.014 *$ \\
\hline \multicolumn{4}{|l|}{ Vascular risk factors, $n(\%)$} \\
\hline Diabetes mellitus & $46(30.1)$ & $37(24.2)$ & 0.304 \\
\hline Smoking & $61(39.9)$ & $62(40.5)$ & 1.000 \\
\hline Hypertension & $126(82.4)$ & $121(79.1)$ & 0.562 \\
\hline Dyslipidemia & $101(66.0)$ & $74(48.4)$ & $0.003 *$ \\
\hline \multicolumn{4}{|l|}{ Medical history, $n(\%)$} \\
\hline CAD & $68(44.4)$ & $56(36.6)$ & 0.200 \\
\hline Atrial fibrillation & $24(15.7)$ & $39(25.5)$ & $0.047 *$ \\
\hline PAOD & $13(8.5)$ & $19(12.4)$ & 0.350 \\
\hline CAOD & $11(7.2)$ & $8(5.2)$ & 0.637 \\
\hline Stroke & $13(8.5)$ & $12(7.8)$ & 1.000 \\
\hline COPD & $18(11.8)$ & $32(20.9)$ & $0.044^{*}$ \\
\hline \multicolumn{4}{|l|}{ Laboratory findings } \\
\hline Trop pos., $n(\%)$ & $73(47.7)$ & $74(48.4)$ & 1.000 \\
\hline Creatinine, $\mathrm{mg} / \mathrm{dl}$ & $1.08(0.93-1.31)$ & $1.06(0.84-1.28)$ & 0.056 \\
\hline $\mathrm{GFR}<60 \mathrm{ml} / \mathrm{min} / 1.73 \mathrm{~m}^{2}, n(\%)$ & $55(35.9)$ & $62(40.5)$ & 0.480 \\
\hline $\mathrm{Hb}, \mathrm{g} / \mathrm{dl}$ & $13.62( \pm 2.05)$ & $10.83( \pm 1.79)$ & $<0.001^{*}$ \\
\hline Platelet count,/nl & $236(192-277)$ & $219(169-281)$ & 0.060 \\
\hline Reduced EF $(<40 \%), n(\%)$ & $16(12.3)$ & $27(18.8)$ & 0.183 \\
\hline \multicolumn{4}{|l|}{ Medication history, $n(\%)$} \\
\hline Acetylsalicylic acid & $99(64.7)$ & $70(45.8)$ & $0.001 *$ \\
\hline Dual antiplatelet therapy & $17(11.1)$ & $15(9.8)$ & 0.852 \\
\hline Anticoagulation & $16(10.5)$ & $14(9.2)$ & 0.848 \\
\hline Statin use & $75(49.0)$ & $44(28.8)$ & $<0.001^{*}$ \\
\hline Beta-blockers & $82(53.6)$ & $97(63.4)$ & 0.104 \\
\hline
\end{tabular}

$B M I$ body mass index, $C A D$ coronary artery disease, $P A O D$ peripheral artery occlusive disease, $C A O D$ cerebral artery occlusive disease, $C O P D$ chronic obstructive pulmonary disease, Trop pos. Troponin positive, $G F R$ glomerular filtration rate, $H b$ hemoglobin, $E F$ ejection fraction

Data are shown as mean $( \pm \mathrm{SD})$ or median (IQR)

*Statistically significant difference between non-cancer and cancer cohort 


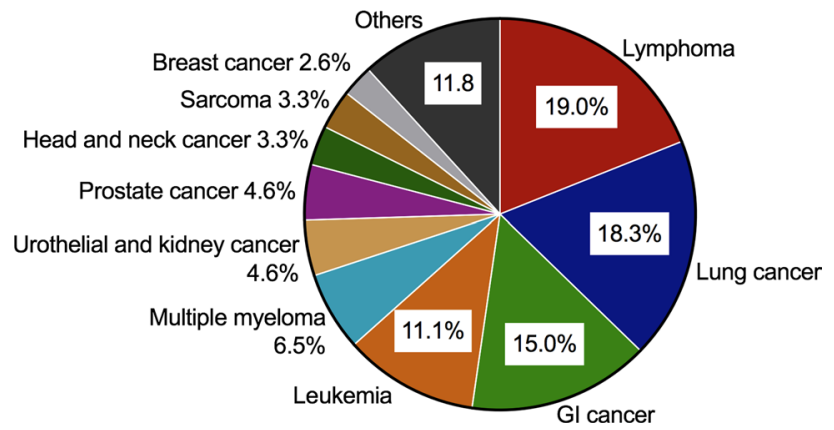

Fig. 2 Differentiation of the study population by cancer entity. Lymphoma was the most common cancer entity with 29 patients (19\%), followed by 28 patients with lung cancer (18.3\%), 23 patients with gastrointestinal (GI) cancer (15\%), and 17 patients with leukemia $(11.1 \%)$

differences between the groups. Multivessel disease, defined as 2- or 3-vessel-disease, was found in $58.8 \%$ of the non-cancer and in $52.9 \%$ of the cancer patients. Rates of primary PCI are presented also in Fig. 3b. Cancer patients received a primary PCI less frequently ( $40.5 \%$ vs. $53.6 \%$, cancer vs. noncancer patients, $p=0.029)$. This difference could be traced back to patients presenting with NSTEMI (PCI rate $40.4 \%$ in cancer patients and $63.5 \%$ in non-cancer patients, $p=0.015$ ), whereas PCI rate in patients with STEMI, unstable, or stable angina did not significantly differ between cancer and non-cancer groups. Multivariable logistic regression analysis identified especially dyslipidemia as a significant predictive factor for receiving a primary PCI in the cancer cohort (OR [95\% CI]: 2.69 [1.211-5.973], $p=0.015$ ), whereas, for example, smoking did not constitute a predictive factor in this context (0.906 [0.433-1.897], $p=0.794)$. For non-cancer patients, both dyslipidemia and smoking represent predictive factor for receiving a primary PCI (2.281 [1.074-4.845], $p=0.032$ and 2.363 [1.147-4.868], $p=0.02$, respectively). The presence of a multivessel CAD was detected more often in cancer patients with previously known CAD (12.9 [4.647-35.813], $p<0.001)$ and in non-cancer patients with diabetes (6.221 [2.099-18.435], $p=0.001)$, dyslipidemia (4.148 [1.691-10.172], $p=0.002)$, and also with previously known CAD (6.055 [2.509-14.612], $p<0.001)$. Regarding cancer history and therapy, the categories in univariate analysis (radiation, chemotherapy, anthracyclines, 5-fluorouracil, surgery, metastatic cancer, and cancer entity) had no significant impact on PCI rates (Supplemental Figure 1).
Table 2 Results of cardiac catheter examinations

\begin{tabular}{lccc}
\hline & Non-cancer $(n=153)$ & Cancer $(n=153)$ & $p$ value \\
\hline Findings, $n(\%)$ & & & \\
Multivessel disease & $90(58.8)$ & $81(52.9)$ & 0.646 \\
Absence of CAD & $40(26.1)$ & $44(28.8)$ & 0.205 \\
CTO & $31(10.3)$ & $29(19.0)$ & 0.886 \\
LMCA stenosis & $10(6.5)$ & $18(11.8)$ & 0.164 \\
Culprit lesion & $84(54.9)$ & $74(48.4)$ & 0.303 \\
Small vessel disease & $68(44.4)$ & $65(42.5)$ & 0.818 \\
PCI (any) & $82(53.6)$ & $62(40.5)$ & $0.029^{*}$ \\
DES & $76(49.7)$ & $38(24.8)$ & $<0.001^{*}$ \\
BMS & $1(0.7)$ & $24(15.7)$ & $<0.001^{*}$ \\
SYNTAX-score & & \\
Baseline-score & $6(0-13)$ & $4(0-12)$ & 0.391 \\
Lowest tertile $(\leq 22), n(\%)$ & $127(90.7)$ & $122(90.3)$ & 1.000 \\
Intermediate tertile $(23-32)$ & $7(5.0)$ & $9(6.7)$ & 0.613 \\
Highest tertile $(\geq 33)$ & $6(4.3)$ & $4(3.0)$ & 0.750 \\
Score post-PCI & $0(0-6)$ & $0(0-5)$ & 0.578 \\
Residual score $>8, n(\%)$ & $29(20.7)$ & $20(14.8)$ & 0.212 \\
LVEDP ${ }^{\S}$, mmHg & $16(12-20.75)$ & $14(8-20)$ & 0.084 \\
\hline
\end{tabular}

$C A D$ coronary artery disease, $C T O$ chronic total occlusion, $L M C A=$ left main coronary artery, $P C I$ percutaneous coronary intervention, DES drug-eluting stent, BMS bare-metal stent, SYNTAX SYNergy between PCI with TAXus and cardiac surgery, LVEDP left-ventricular end-diastolic pressure

Data are shown as median (IQR)

* Statistically significant difference between non-cancer and cancer cohort

${ }^{\dagger}$ Excluded patients with history for coronary artery bypass surgery, included: cancer $n=135$, non-cancer $n=140$

Patients with performed PCI: cancer $n=57$, non-cancer $n=73$

${ }^{\S}$ Documented LVEDP: cancer $n=110$, non-cancer $n=102$ 


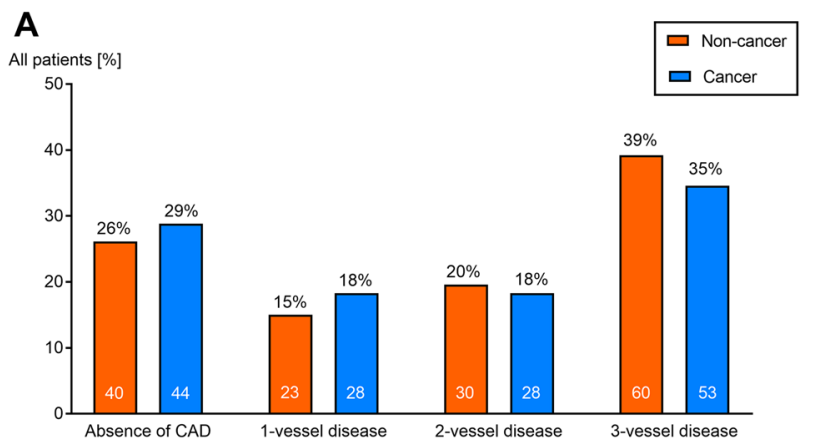

B
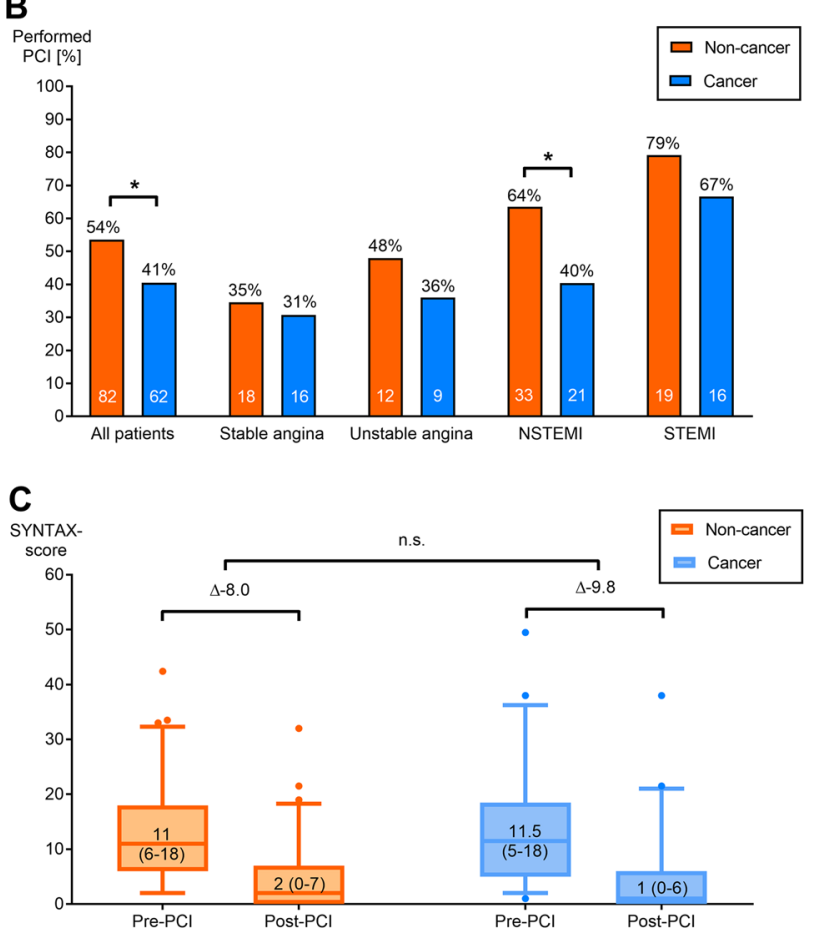

Fig. 3 Results of cardiac catheter examinations. Analysis of coronary angiography results to distinguish between in 1-, 2-, and 3-vessel-disease was conducted. Occurrence of coronary artery disease (CAD) was similar in non-cancer and cancer patients (a). Rate of PCI related to clinical presentation showing a significant difference in non-cancer vs. cancer patients [all patients, $(p=0.029)$ ], particularly with non-ST-segment elevation myocardial infarction (NSTEMI); $p=0.015$ (b). Evaluation of coronary angiography results were conducted according to SYNTAX (SYNergy between PCI with TAXus and cardiac surgery)-score calculation. Patients with coronary artery bypasses were excluded. Improvement of SYNTAX-score after percutaneous coronary intervention (PCI) is illustrated before (pre-) and after (post-) PCI for non-cancer and cancer cohort ( $p=0.2$, not significant (n.s.)) (c)

Interestingly, multivariable analysis identified metastatic cancer and anthracycline chemotherapy as positive predictors for receiving a primary PCI (3.265 [1.455-7.325], $p=0.004$ and 3.139 [1.228-8.027], $p=0.017)$. Treatment with 5-fluorouracil did not influence PCI rate significantly (0.425 [0.157-1.152], $p=0.093)$. From 27 patients with 5-fluorouracil treatment, ten patients had no obstructive coronary artery disease (37\%), which is not significantly different compared to all included cancer patients $(p=0.650)$. Vasospasm, which is a typical 5-fluorouracil side effect, was suspected in only one case. Table 3 summarizes the results of cardiac catheter examination depending on chemotherapy with anthracyclines, 5-fluorouracil, and platinum-based or alkylating agents (e.g., cyclophosphamide).

Detailed evaluation of coronary angiography results was conducted according to the SYNTAX-scoring system (Table 2). Patients with coronary artery bypasses were excluded [25]. Baseline and proportion with a residual SYNTAX-score $>8$ (defining an incomplete revascularization [26]) did not significantly differ between cancer and non-cancer patients. Change of SYNTAX-score upon PCI is depicted in Fig. 3c. No significant differences between cancer and control groups were found regarding pre-PCI and post-PCI SYNTAX-score ( $n=73$ non-cancer patients, $n=57$ cancer patients). Delta between pre- and post-PCI SYNTAXscore as a marker for the PCI result was similar (non-cancer patients -8.0 , cancer patients -9.8 points, $p=0.2$ ). Leftventricular end-diastolic pressure as a marker for coronary microvascular disease was also similar in cancer cohort vs. non-cancer cohort (Table 2). However, differences were detected regarding peri-procedural device application: cancer patients received bare-metal stents more frequently $(p<0.001)$ and fewer drug-eluting stents $(p<0.001)$ compared to control group (Table 2).

Hemoglobin value was lower in cancer patients before coronary angiography $(10.83 \pm 1.79 \mathrm{~g} / \mathrm{dl}$ vs. $13.62 \pm 2.05 \mathrm{~g} /$ $\mathrm{dl}, p<0.001$ ), but hemoglobin drop within the first $48 \mathrm{~h}$ after coronary intervention did not differ significantly (median [IQR]: 0.55 [0.0-1.08] g/dl vs. 0.6 [0.0-1.40] g/dl, $p=0.329)$. Cancer patients received red blood cell transfusion more often $(9.2 \%$ vs. $1.3 \%, p=0.003)$, which was not linked to the occurrence of increased post-interventional bleeding or hematoma. Referring to the BARC definition of peri-interventional bleeding complications, hemoglobin drop between 3 and $5 \mathrm{~g} / \mathrm{dl}$ (BARC 3a) occurred two times and $>5 \mathrm{~g} / \mathrm{dl}$ (BARC $3 \mathrm{~b}$ ) once in both, cancer and control, groups. Surgical intervention (BARC 3b) was necessary once within the cancer group due to a femoral dissection. In-hospital mortality and 30-day mortality were not significantly different in cancer and non-cancer patients (HR 1.2 [0.51-2.76], $p=0.68$ ), which indicates that acute complications or early cardiovascular events after cardiac catheterization (e.g., fatal bleeding and re-infarct rate) leading to death are similar in the two groups.

All-cause mortality was analyzed using Kaplan-Meier procedure with a follow-up of 1 year showing a higher mortality in cancer patients compared to non-cancer patients (HR 5.5 [3.0-10.2], $p<0.001$, Fig. 4a). Multivariable regression analysis identified male sex (0.286 [0.116-0.705], $p=0.007)$, 
Table 3 Results of cardiac catheter examinations depending on cancer therapy

\begin{tabular}{|c|c|c|c|c|c|}
\hline & Anthracyclines $(n=35)$ & 5-Fluorouracil $(n=27)$ & Platinum-based $(n=40)$ & Alkylating agents $(n=26)$ & $\begin{array}{l}\text { All cancer } \\
\text { patients } \\
(n=153)\end{array}$ \\
\hline \multicolumn{6}{|l|}{ Findings, $n(\%)$} \\
\hline Multivessel disease & $16(45.7)$ & $12(44.4)$ & $20(50.0)$ & $11(42.3)$ & $81(52.9)$ \\
\hline Absence of CAD & $11(31.4)$ & $10(37.0)$ & $11(27.5)$ & $10(38.5)$ & $44(28.8)$ \\
\hline СТO & $6(17.1)$ & $3(11.1)$ & $7(17.5)$ & $3(11.5)$ & $29(19.0)$ \\
\hline LMCA stenosis & $5(14.3)$ & $2(7.4)$ & $2(5.0)$ & $3(11.5)$ & $18(11.8)$ \\
\hline Culprit lesion & $19(54.3)$ & $13(48.1)$ & $21(52.5$ & $12(46.2)$ & $74(48.4)$ \\
\hline Small vessel disease & $14(40.0)$ & $9(33.3)$ & $20(50.0)$ & $15(57.7)$ & $65(42.5)$ \\
\hline PCI (any) & $16(45.7)$ & 8 (29.6) & $19(47.5)$ & $13(50.0)$ & $62(40.5)$ \\
\hline DES & $6(17.1)$ & $4(14.8)$ & $12(30.0)$ & $6(23.1)$ & $38(24.8)$ \\
\hline BMS & $10(28.6)^{*}$ & $4(14.8)$ & $7(17.5)$ & $7(26.9)$ & $24(15.7)$ \\
\hline \multicolumn{6}{|l|}{ SYNTAX-score ${ }^{\dagger}$} \\
\hline Baseline-Score & $3(0.0-7.75)$ & $3.5(0.0-7.75)$ & $5(0.0-13.88)$ & $2(0.0-7.0)$ & $4(0-12)$ \\
\hline LVEDP $^{\S}, \mathrm{mmHg}$ & $10(7.0-15.0)$ & $10(7.25-15.5)$ & $12(7.25-22.25)$ & $14.5(7.75-22.75)$ & $14(8-20)$ \\
\hline
\end{tabular}

$C A D$ coronary artery disease, $C T O$ chronic total occlusion, $L M C A=$ left main coronary artery, $P C I$ percutaneous coronary intervention, $D E S$ drug-eluting stent, BMS bare-metal stent, SYNTAX SYNergy between PCI with TAXus and cardiac surgery, LVEDP left-ventricular end-diastolic pressure

Data are shown as median (IQR)

*Statistically significant difference between cancer patients under anthracycline therapy and all cancer patients

$\dagger$ excluded patients with history for coronary artery bypass surgery

$\S$ patients with documented LVEDP

troponin-positive ACS (2.365 [1.162-4.817], $p=0.018)$, and lower hemoglobin value (0.719 [0.575-0.898], $p=0.004)$ as predictors for 1-year mortality in cancer cohort. In noncancer patients, none of the analyzed factors predicted 1-year mortality. Using Kaplan-Meier procedure, cancer patients with troponin-positive ACS (STEMI and NSTEMI) showed significantly higher 5-year mortality compared to cancer patients with troponin-negative angina (HR 1.75 [1.18-2.6], $p=0.005$, Fig. 4b). Subgroup analysis between advanced cancer and non-advanced cancer did not abrogate this effect, showing the highest 5-year mortality in patients with advanced cancer and troponin-positive ACS (HR 1.59 [1.07-2.38], $p=0.023$, Supplemental Figure 2). Advanced cancer was defined as metastatic cancer or depending on tumor stage (UICC stage III/IV, Ann-Arbor stage IV, Durie and Salmon stage 3, TMN stadium > T2b). Baseline characteristic of cancer patients with troponin-positive ACS vs. troponin-negative angina did not significantly differ (Supplemental Table 1), whereas results of cardiac catheter examinations documented a higher SYNTAX-score at baseline and a higher rate for PCI in cancer patients with troponinpositive ACS (Supplemental Table 2). To better classify the mortality rate of our study patients, we compared these with the 5-year survival of cancer patients from the US National Cancer Institute registry (SEER) [27]. Calculated 5-year survival rate based on SEER data would be $42.4 \%$, which is not significantly higher compared to overall survival of $34 \%$ in our cancer cohort ( $p=0.158$, Fig. $4 b$ ). However, 5 -year survival rate of cancer patients with troponin-positive ACS was with $28.9 \%$ significantly lower than in the calculated SEER cancer group $(p=0.017)$. This indicates that performing a coronary angiography in itself does not increase mortality in cancer patients, but the occurrence of a troponin-positive acute coronary syndrome may be associated with a higher mortality.

\section{Discussion}

The concurrent incidence of cancer and CAD relates to overlapping risk factors, side effects of cancer therapy, and negative cardiovascular effects of cancer itself $[4,28]$. Because of the high morbidity and mortality, cancer is often considered as a contraindication for coronary intervention [16, 17]. Patients with discharge diagnosis of STEMI or NSTEMI from a single-center assessment between 2000 and 2006 showed a remarkably low catheter-based revascularization rate of only $3.3 \%$, because non-interventional therapy is often preferred in cancer patients [17]. In this study, we included patients assessed by coronary angiography during intra-hospital cancer treatment and conducted a comparison to a matched control cohort. The severity of CAD defined by the SYNTAX-score and distribution in 1-, 2-, or 3-vesseldisease did not differ significantly in cancer and non-cancer. 

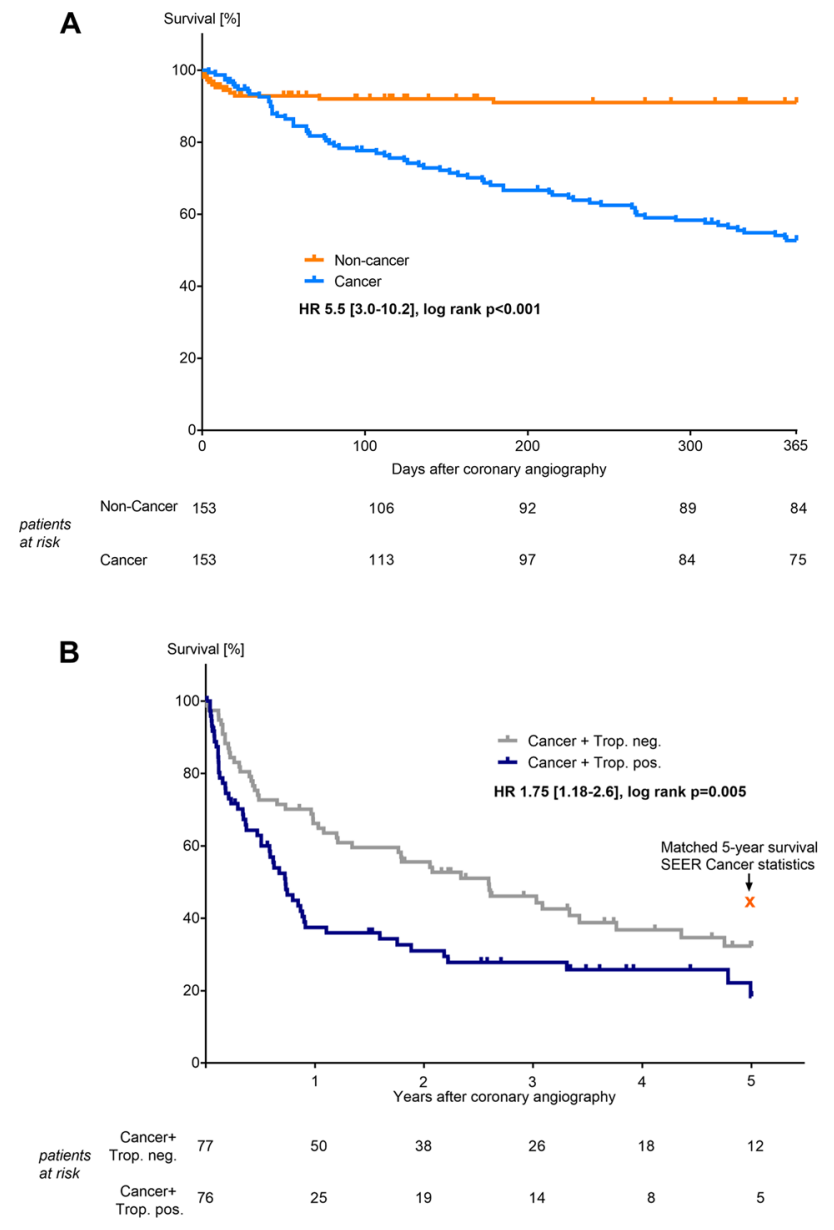

Fig. 4 Kaplan-Meier analyses. One-year all-cause mortality of cancer patients was significantly higher compared to non-cancer patients, log-rank (Mantel Cox) $p<0.001$. Mortality after 30 days was similar, log-rank (Mantel Cox) $p=0.68$ (a). Five-year all-cause mortality of cancer patients with troponin-positive acute coronary syndrome (trop. pos./cancer patients with STEMI and NSTEMI) was significantly higher compared to cancer patients with troponin-negative angina (trop. neg./cancer patients with unstable and stable angina), log-rank (Mantel Cox) $p=0.005$. Matched 5-year survival of cancer patients from the US National Cancer Institute Surveillance, Epidemiology, and End Results Program (SEER) was $42.4 \%$ (b)

However, the rate of PCI was lower in cancer patients, especially in patients with an NSTEMI diagnosis.

Our study confirms the results of previous reports, which highlighted a potentially insufficient treatment of patients with cancer and CAD from the clinical and interventional point of view [16, 17, 29-31] that was less adherent to American and European Guidelines [5, 6, 32]. Bare-metal stents have been preferred in cancer patients [18,33], whereas data in general population unequivocally favor drug-eluting stents $[34,35]$. This could have changed due implementation of new guidelines which no longer recommend these devices (31) and propose the same duration of dual antiplatelet therapy for 6 month for stable angina/chronic coronary syndromes and 12 months for ACS (for patients without indication for oral anticoagulation) for drug-eluting stents or drug-coated balloons [36]. However, detailed evidence for device selection and antiplatelet therapy is scarce. Interestingly, a large data analysis from the National Inpatient Sample for Hospital Discharges in the United States of America between 2004 and 2014 showed better outcomes in cancer patients and PCI when treated with drug-eluting stents rather than a bare-metal stents [31]. Bleeding complications are feared in cancer patients also because of lower hemoglobin values and platelet counts before coronary angiography. Application and duration of dual antiplatelet therapy pose a challenge, too, because cut-off values of low thrombocyte levels under cancer and cancer therapy do not exist coherently [30]. Additionally, thromboembolic events are frequently encountered in cancer patients, which can be treated by low-molecular-weight heparin [37] or oral anticoagulation with an acceptable incidence of major bleedings [38-41], but this complicates the therapeutic management in context of coronary angiography, PCI, and antiplatelet therapy. In-hospital and 30-day all-cause mortality as well as documented catheter-associated bleeding complications did not differ between cancer and non-cancer groups, which suggests a comparable peri-procedural risk in our study cohort.

In recent years, advances in tumor therapy lead to an improved survival of cancer patients [2, 42] and, therefore, it is important to emphasize the management of comorbidities and treatment-related cardiovascular toxicities [7, 43]. This also includes optimal primary and secondary prevention of cardiovascular diseases in cancer patients. The importance for early and intensive modification of cardiovascular risk factors was also highlighted in the last clinical practice guidelines from the American Society of Clinical Oncology for the treatment of breast cancer patients [44]. In our study population, previous treatment with acetylsalicylic acid and lipid-lowering therapy with statins was significantly less frequent in the cancer group, although there were no differences regarding patient's medical history of known CAD and cardiovascular risk factors between cancer and control group.

The present study has several limitations. This study was retrospective and cancer patients were searched from the inpatient hospital database. Time interval between performed cancer therapy, whether chemotherapy or radiation, was not analyzed. Also, therapy protocols differ severely between the cancer patients. Overall, our study population is a mixed cohort but, therefore, representative for clinical practice. Differences between the cancer and non-cancer groups could have been influenced by different time periods of performed coronary angiography. Cancer patients were included between 2011 and 2018, whereas matched control patients received coronary angiography between 2017 and 
2018. Management of patients with ACS or stable angina as well as myocardial revascularization strategies could be changed due to renewed practice guidelines $[6,9]$. This, however, would not apply to the increased mortality in the cancer group itself. Selection of inpatient cancer patients comes along with high morbidity and mortality [45]. More than half of all cancer deaths occurred in an acute care hospital [46]. This leads to a comparably high 5-year mortality in our cancer cohort (66\%), especially in patients with troponin-positive ACS (71\%). This was also true in others studies with a 1-year survival as low as $26 \%$ of hospitalized cancer patients with ACS [17]. Another study documented a significant greater in-hospital mortality of patients with lung cancer undergoing PCI [31], which was one of the most common cancer entities in our study population. Our data only provide information of all-cause mortality. Determining the cause of death is usually difficult in cancer patients due to a complex clinical picture of existing diseases, especially within the last weeks before death. Often palliative care is needed because of pain medication and sedation. Clinical studies and publications dealing with this topic are rare. Different cancer entities seem to be associated with different causes of death [47]. Multiorgan failure, cardiovascular diseases, cachexia, and severe infection are some of the most named causes of death in cancer patients [48, 49]. To avoid any classification bias, we focused on all-cause mortality in this study.

\section{Conclusions}

Hospitalized cancer patients were undertreated regarding performed PCI, particularly patients with troponin-positive ACS which additionally showed the highest mortality rate. SYNTAX-scores suggest a good interventional result in cancer patients if PCI was performed. This underlines the importance of further characterization and recommendation for clinical behavior and treatment of these patients. Initial management of cancer patients with elevated troponin or angina pectoris symptoms remains difficult in daily clinical practice. Identification of cardiovascular risk factors, and primary and secondary prevention are highly important especially in cancer patients. Cardio-oncology is a multidisciplinary field with increasing patient numbers and, therefore, poses a challenge to the medical system.

\section{Acknowledgments Open Access funding provided by Projekt DEAL.}

Funding This work was supported by the IFORES research grant from the Medical Faculty, University Duisburg-Essen, Hufelandstraße 55, 45147 Essen, Germany (S. M. Mrotzek).
Availability of data and materials The datasets generated and/or analyzed during the current study are available from the corresponding author upon reasonable request.

Conflicts of interest S D. Anker has received research support from Abbott Vascular and Vifor, and reports personal fees from Abbott, Actimed, Bayer, Boehringer Ingelheim, Novartis, Servier, and Vifor. M. $\mathrm{S}$. Anker has received research support from the German Cardiovascular Research Center and personal fees from Servier. All other authors reported no relevant disclosures.

Ethical standards All procedures were in accordance with the ethical standards of the institutional ethics committees of the participating centers and with the 1964 Helsinki Declaration and its later amendments or comparable ethical standards.

Open Access This article is licensed under a Creative Commons Attribution 4.0 International License, which permits use, sharing, adaptation, distribution and reproduction in any medium or format, as long as you give appropriate credit to the original author(s) and the source, provide a link to the Creative Commons licence, and indicate if changes were made. The images or other third party material in this article are included in the article's Creative Commons licence, unless indicated otherwise in a credit line to the material. If material is not included in the article's Creative Commons licence and your intended use is not permitted by statutory regulation or exceeds the permitted use, you will need to obtain permission directly from the copyright holder. To view a copy of this licence, visit http://creativecommons.org/licenses/by/4.0/.

\section{References}

1. Iannaccone M, D'Ascenzo F, Vadala P, Wilton SB, Noussan P, Colombo F et al (2017) Prevalence and outcome of patients with cancer and acute coronary syndrome undergoing percutaneous coronary intervention: a BleeMACS substudy. Eur Heart J Acute Cardiovasc Care. https://doi.org/10.1177/2048872617706501

2. Yeh ET, Chang HM (2016) Oncocardiology-past, present, and future: a review. JAMA Cardiol 1(9):1066-1072. https://doi. org/10.1001/jamacardio.2016.2132

3. Totzeck M, Schuler M, Stuschke M, Heusch G, Rassaf T (2019) Cardio-oncology_-strategies for management of cancer-therapy related cardiovascular disease. Int J Cardiol 280:163-175. https ://doi.org/10.1016/j.ijcard.2019.01.038

4. Al-Kindi SG, Oliveira GH (2016) Prevalence of preexisting cardiovascular disease in patients with different types of cancer: the unmet need for onco-cardiology. Mayo Clin Proc 91(1):81-83. https://doi.org/10.1016/j.mayocp.2015.09.009

5. Michel L, Rassaf T (2019) Cardio-oncology: need for novel structures. Eur J Med Res 24(1):1. https://doi.org/10.1186/s4000 1-018-0359-0

6. Fihn SD, Blankenship JC, Alexander KP, Bittl JA, Byrne JG, Fletcher BJ et al (2014) 2014 ACC/AHA/AATS/PCNA/SCAI/ STS focused update of the guideline for the diagnosis and management of patients with stable ischemic heart disease: a report of the American College of Cardiology/American Heart Association Task Force on Practice Guidelines, and the American Association for Thoracic Surgery, Preventive Cardiovascular Nurses Association, Society for Cardiovascular Angiography and Interventions, and Society of Thoracic Surgeons. J Am Coll Cardiol 64(18):1929-1949. https://doi.org/10.1016/j.jacc.2014.07.017

7. Rassaf T, Totzeck M, Backs J, Bokemeyer C, Hallek M, HilfikerKleiner D et al (2020) Onco-cardiology: consensus paper of the 
german cardiac society, the german society for pediatric cardiology and congenital heart defects and the german society for hematology and medical oncology. Clin Res Cardiol. https://doi. org/10.1007/s00392-020-01636-7

8. Michel L, Rassaf T, Totzeck M (2018) Biomarkers for the detection of apparent and subclinical cancer therapy-related cardiotoxicity. J Thorac Dis 10:S4282-S4295. https://doi.org/10.21037 /jtd.2018.08.15

9. Amsterdam EA, Wenger NK, Brindis RG, Casey DE Jr, Ganiats TG, Holmes DR Jr et al (2014) 2014 AHA/ACC guideline for the management of patients with non-ST-elevation acute coronary syndromes: executive summary: a report of the American College of Cardiology/American Heart Association Task Force on Practice Guidelines. Circulation 130(25):2354-2394. https://doi. org/10.1161/CIR.0000000000000133

10. Cardinale D, Sandri MT, Colombo A, Colombo N, Boeri M, Lamantia G et al (2004) Prognostic value of troponin I in cardiac risk stratification of cancer patients undergoing high-dose chemotherapy. Circulation 109(22):2749-2754. https://doi. org/10.1161/01.CIR.0000130926.51766.CC

11. Myocardial infarction redefined-a consensus document of The Joint European Society of Cardiology/American College of Cardiology Committee for the redefinition of myocardial infarction. (2000) Eur Heart J 21(18):1502-13. https://doi.org/10.1053/ euhj.2000.2305

12. Cardinale D, Sandri MT, Martinoni A, Tricca A, Civelli M, Lamantia $G$ et al (2000) Left ventricular dysfunction predicted by early troponin I release after high-dose chemotherapy. J Am Coll Cardiol 36(2):517-522

13. Cardinale D, Sandri MT, Martinoni A, Borghini E, Civelli M, Lamantia $G$ et al (2002) Myocardial injury revealed by plasma troponin I in breast cancer treated with high-dose chemotherapy. Ann Oncol 13(5):710-715

14. Michel L, Mincu RI, Mrotzek SM, Korste S, Neudorf U, Rassaf T et al (2020) Cardiac biomarkers for the detection of cardiotoxicity in childhood cancer-a meta-analysis. ESC Heart Fail 7(2):423433. https://doi.org/10.1002/ehf2.12589

15. Michel L, Mincu RI, Mahabadi AA, Settelmeier S, Al-Rashid F, Rassaf $T$ et al (2020) Troponins and brain natriuretic peptides for the prediction of cardiotoxicity in cancer patients: a meta-analysis. Eur J Heart Fail 22(2):350-361. https://doi.org/10.1002/ejhf.1631

16. Iannaccone $\mathrm{M}$ et al (2017) Optimal medical therapy in patients with malignancy undergoing percutaneous coronary intervention for acute coronary syndrome: a BleeMACS Sub-Study. Am J Cardiovasc Drugs 17(1):61-71. https://doi.org/10.1007/s4025 6-016-0196-x

17. Yusuf SW, Daraban N, Abbasi N, Lei X, Durand JB, Daher IN (2012) Treatment and outcomes of acute coronary syndrome in the cancer population. Clin Cardiol 35(7):443-450. https://doi. org/10.1002/clc. 22007

18. Wang F, Gulati R, Lennon RJ, Lewis BR, Park J, Sandhu GS et al (2016) Cancer History Portends Worse Acute and Long-term Noncardiac (but Not Cardiac) mortality after primary percutaneous coronary intervention for acute ST-segment elevation myocardial infarction. Mayo Clin Proc 91(12):1680-1692. https://doi. org/10.1016/j.mayocp.2016.06.029

19. Iliescu C, Durand JB, Kroll M (2011) Cardiovascular interventions in thrombocytopenic cancer patients. Tex Heart Inst $\mathbf{J}$ 38(3):259-260

20. Mincu RI, Rassaf T, Totzeck M (2018) Red blood cell transfusion in patients with ST-elevation myocardial infarction-a meta-analysis of more than 21,000 patients. Neth Heart J 26(9):454-460. https://doi.org/10.1007/s12471-018-1137-x

21. Chang HM, Moudgil R, Scarabelli T, Okwuosa TM, Yeh ETH (2017) Cardiovascular complications of cancer therapy: best practices in diagnosis, prevention, and management: part $1 . \mathrm{J}$
Am Coll Cardiol 70(20):2536-2551. https://doi.org/10.1016/j. jacc.2017.09.1096

22. Rosenbaum PR, Rubin DB (1985) Constructing a Control-Group Using Multivariate Matched Sampling Methods That Incorporate the Propensity Score. Am Stat 39(1):33-38. https://doi. org/10.2307/2683903

23. Mehran R, Rao SV, Bhatt DL, Gibson CM, Caixeta A, Eikelboom $\mathrm{J}$ et al (2011) Standardized bleeding definitions for cardiovascular clinical trials: a consensus report from the Bleeding Academic Research Consortium. Circulation 123(23):2736-2747. https:// doi.org/10.1161/CIRCULATIONAHA.110.009449

24. Serruys PW, Morice MC, Kappetein AP, Colombo A, Holmes DR, Mack MJ et al (2009) Percutaneous coronary intervention versus coronary-artery bypass grafting for severe coronary artery disease. N Engl J Med 360(10):961-972. https://doi.org/10.1056/NEJMo a0804626

25. Sianos G, Morel MA, Kappetein AP, Morice MC, Colombo A, Dawkins K et al (2005) The SYNTAX Score: an angiographic tool grading the complexity of coronary artery disease. EuroIntervention 1(2):219-227

26. Genereux P, Palmerini T, Caixeta A, Rosner G, Green P, Dressler O et al (2012) Quantification and impact of untreated coronary artery disease after percutaneous coronary intervention: the residual SYNTAX (Synergy Between PCI with Taxus and Cardiac Surgery) score. J Am Coll Cardiol 59(24):21652174. https://doi.org/10.1016/j.jacc.2012.03.010

27. Henley SJ, Ward EM, Scott S, Ma J, Anderson RN, Firth AU et al (2020) Annual report to the nation on the status of cancer, part I: national cancer statistics. Cancer 126(10):2225-2249. https://doi.org/10.1002/cncr.32802

28. Totzeck M, Mincu RI, Rassaf T (2017) Cardiovascular adverse events in patients with cancer treated with bevacizumab: a metaanalysis of more than 20000 patients. J Am Heart Assoc 6:8. https://doi.org/10.1161/jaha.117.006278

29. Landes U, Kornowski R, Bental T, Assali A, Vaknin-Assa H, Lev E et al (2017) Long-term outcomes after percutaneous coronary interventions in cancer survivors. Coron Artery Dis 28(1):5-10. https://doi.org/10.1097/MCA.0000000000000429

30. Banasiak W, Zymlinski R, Undas A (2018) Optimal management of cancer patients with acute coronary syndrome. Pol Arch Intern Med 128(4):244-253. https://doi.org/10.20452/ pamw.4254

31. Potts JE, Iliescu CA, Lopez Mattei JC, Martinez SC, Holmvang L, Ludman P et al (2019) Percutaneous coronary intervention in cancer patients: a report of the prevalence and outcomes in the United States. Eur Heart J 40(22):1790-1800. https://doi.org/10.1093/ eurheartj/ehy 769

32. Roffi M, Patrono C, Collet JP, Mueller C, Valgimigli M, Andreotti F et al (2015) 2015 ESC guidelines for the management of acute coronary syndromes in patients presenting without persistent STsegment Elevation. Rev Esp Cardiol (Engl Ed) 68(12):1125. https ://doi.org/10.1016/j.rec.2015.10.009

33. Al-Kindi SG, Oliveira GH (2016) Onco-Cardiology: a Tale of Interplay Between 2 Families of Diseases. Mayo Clin Proc 91(12):1675-1677. https://doi.org/10.1016/j.mayocp.2016.10.015

34. Neumann FJ, Sousa-Uva M, Ahlsson A, Alfonso F, Banning AP, Benedetto U et al (2019) 2018 ESC/EACTS Guidelines on myocardial revascularization. Eur Heart J 40(2):87-165. https://doi. org/10.1093/eurheartj/ehy394

35. Kastrati A, Dibra A, Spaulding C, Laarman GJ, Menichelli M, Valgimigli M et al (2007) Meta-analysis of randomized trials on drug-eluting stents vs bare-metal stents in patients with acute myocardial infarction. Eur Heart J 28(22):2706-2713. https:// doi.org/10.1093/eurheartj/ehm402

36. Levine GN, Bates ER, Bittl JA, Brindis RG, Fihn SD, Fleisher LA et al (2016) 2016 ACC/AHA Guideline Focused Update on 
Duration of Dual Antiplatelet Therapy in Patients With Coronary Artery Disease: a Report of the American College of Cardiology/American Heart Association Task Force on Clinical Practice Guidelines: An Update of the 2011 ACCF/AHA/SCAI Guideline for Percutaneous Coronary Intervention, 2011 ACCF/AHA Guideline for Coronary Artery Bypass Graft Surgery, 2012 ACC/ AHA/ACP/AATS/PCNA/SCAI/STS Guideline for the Diagnosis and Management of Patients With Stable Ischemic Heart Disease, 2013 ACCF/AHA Guideline for the Management of ST-Elevation Myocardial Infarction, 2014 AHA/ACC Guideline for the Management of Patients With Non-ST-Elevation Acute Coronary Syndromes, and 2014 ACC/AHA Guideline on Perioperative Cardiovascular Evaluation and Management of Patients Undergoing Noncardiac Surgery. Circulation 134(10):e123-e155. https://doi. org/10.1161/CIR.0000000000000404

37. Lee AY, Levine MN, Baker RI, Bowden C, Kakkar AK, Prins M et al (2003) Low-molecular-weight heparin versus a coumarin for the prevention of recurrent venous thromboembolism in patients with cancer. N Engl J Med 349(2):146-153. https://doi. org/10.1056/NEJMoa025313

38. Schulman S, Kearon C, Kakkar AK, Mismetti P, Schellong S, Eriksson $\mathrm{H}$ et al (2009) Dabigatran versus warfarin in the treatment of acute venous thromboembolism. N Engl J Med 361(24):2342-2352. https://doi.org/10.1056/NEJMoa0906598

39. Investigators $\mathrm{E}$, Bauersachs R, Berkowitz SD, Brenner B, Buller HR, Decousus $\mathrm{H}$ et al (2010) Oral rivaroxaban for symptomatic venous thromboembolism. N Engl J Med 363(26):2499-2510. https://doi.org/10.1056/NEJMoa1007903

40. Agnelli G, Buller HR, Cohen A, Curto M, Gallus AS, Johnson $M$ et al (2013) Oral apixaban for the treatment of acute venous thromboembolism. N Engl J Med 369(9):799-808. https://doi. org/10.1056/NEJMoa1302507

41. Hokusai VTEI, Buller HR, Decousus H, Grosso MA, Mercuri M, Middeldorp S et al (2013) Edoxaban versus warfarin for the treatment of symptomatic venous thromboembolism. N Engl J Med 369(15):1406-1415. https://doi.org/10.1056/NEJMoa1306638
42. Totzeck M, Mincu RI, Mrotzek S, Schadendorf D, Rassaf T (2018) Cardiovascular diseases in patients receiving small molecules with anti-vascular endothelial growth factor activity: a meta-analysis of approximately 29,000 cancer patients. Eur J Prev Cardiol 25(5):482-494. https://doi.org/10.1177/2047487318755193

43. Rassaf T, Totzeck M (2018) Modern concepts in cardio-oncology. J Thorac Dis 10:S4386-S4390. https://doi.org/10.21037/ jtd.2018.11.110

44. Runowicz CD, Leach CR, Henry NL, Henry KS, Mackey HT, Cowens-Alvarado RL et al (2016) American cancer society/american society of clinical oncology breast cancer survivorship care guideline. J Clin Oncol 34(6):611-635. https://doi.org/10.1200/ JCO.2015.64.3809

45. Pfister DG, Rubin DM, Elkin EB, Neill US, Duck E, Radzyner $M$ et al (2015) Risk adjusting survival outcomes in hospitals that treat patients with cancer without information on cancer stage. JAMA Oncol 1(9):1303-1310. https://doi.org/10.1001/jamao ncol.2015.3151

46. DeCaria K, Dudgeon D, Green E, Shaw Moxam R, Rahal R, Niu J et al (2017) Acute care hospitalization near the end of life for cancer patients who die in hospital in Canada. Curr Oncol 24(4):256-261. https://doi.org/10.3747/co.24.3704

47. Brown BW, Brauner C, Minnotte MC (1993) Noncancer deaths in white adult cancer patients. J Natl Cancer Inst 85(12):979-987. https://doi.org/10.1093/jnci/85.12.979

48. Inagaki J, Rodriguez V, Bodey GP (1974) Proceedings: causes of death in cancer patients. Cancer 33(2):568-573. https://doi. org/10.1002/1097-0142(197402)33:2\%3c568:aid-cncr282033 0236\%3e3.0.co;2-2

49. Anker MS, Ebner N, Hildebrandt B, Springer J, Sinn M, Riess $\mathrm{H}$ et al (2016) Resting heart rate is an independent predictor of death in patients with colorectal, pancreatic, and non-small cell lung cancer: results of a prospective cardiovascular long-term study. Eur J Heart Fail 18(12):1524-1534. https://doi.org/10.1002/ ejhf.670

\section{Affiliations}

\section{Simone M. Mrotzek ${ }^{1}$. Alessia Lena ${ }^{2,3,4,5} \cdot$ Sara Hadzibegovic ${ }^{2,3,4,5} \cdot$ Ria Ludwig $^{1} \cdot$ Fadi Al-Rashid $^{1}$ - Amir A. Mahabadi ${ }^{1}$. Raluca I. Mincu ${ }^{1}$ - Lars Michel ${ }^{1}$ - Laura Johannsen ${ }^{1}$ - Lena Hinrichs ${ }^{1}$ - Martin Schuler ${ }^{6,7}$. Ulrich Keller ${ }^{8,9,10}$. Stefan D. Anker ${ }^{2,4,5}$. Ulf Landmesser ${ }^{3,4,5}$. Tienush Rassaf ${ }^{1} \cdot$ Markus S. Anker ${ }^{2,3,4,5}$ - Matthias Totzeck ${ }^{1}$}

\section{Matthias Totzeck}

Matthias.Totzeck@uk-essen.de

1 Department of Cardiology and Vascular Medicine, West German Heart and Vascular Center, Medical Faculty, University Hospital Essen, Hufelandstr. 55, 45147 Essen, Germany

2 Division of Cardiology and Metabolism, Department of Cardiology, Charité Campus Virchow Klinikum (CVK), Berlin, Germany

3 Department of Cardiology, Charité Campus Benjamin Franklin (CBF), Berlin, Germany

4 Berlin Institute of Health Center for Regenerative Therapies (BCRT), Berlin, Germany

5 DZHK (German Center for Cardiovascular Research), Partner Site, Berlin, Germany
6 Department of Medical Oncology, Medical Faculty, West German Cancer Center, University Hospital Essen, Hufelandstr. 55, 45147 Essen, Germany

7 German Cancer Consortium (DKTK), Partner Site University Hospital Essen, Hufelandstrasse 55, 45147 Essen, Germany

8 Department of Hematology, Oncology and Tumor Immunology, Charité Campus Benjamin Franklin (CBF), Berlin, Germany

9 German Cancer Consortium (DKTK), Partner Site, Berlin, Germany

10 Max-Delbrück Center for Molecular Medicine, Berlin, Germany 